\title{
Role of expertise and contralateral symmetry in the diagnosis of Pneumoconiosis: An experimental study
}

\author{
Jampani Varun1a, Vaidya Vivekb, Sivaswamy Jayanthia, L. Tourani Kishore ${ }^{c}$ \\ ${ }^{\mathrm{a} C}$ CVIT, IIIT-Hyderabad, Gachibowli, Hyderabad, AP, INDIA-500 032 \\ ${ }^{\mathrm{b}}$ Imaging Technology Lab, GE Global Research, JFWTC, Bangalore, KA, INDIA- 560066 \\ ${ }^{\mathrm{c}}$ Radiology Department, Care Hospitals, Banjara Hills, Hyderabad, AP, INDIA- 500034
}

\begin{abstract}
Pneumoconiosis, a lung disease caused by the inhalation of dust, is mainly diagnosed using chest radiographs. The effects of using contralateral symmetric (CS) information present in chest radiographs in the diagnosis of pneumoconiosis are studied using an eye tracking experimental study. The role of expertise and the influence of CS information on the performance of readers with different expertise level are also of interest. Experimental subjects ranging from novices \& medical students to staff radiologists were presented with 17 double and 16 single lung images, and were asked to give profusion ratings for each lung zone. Eye movements and the time for their diagnosis were also recorded. Kruskal-Wallis test $(\chi 2(6)=13.38, \mathrm{p}=.038)$, showed that the observer error (average sum of absolute differences) in double lung images differed significantly across the different expertise categories when considering all the participants. Wilcoxon-signed rank test indicated that the observer error was significantly higher for single-lung images $(Z=3.13, p<.001)$ than for the double-lung images for all the participants. Mann-Whitney test $(U=28, p=$ .038) showed that the differential error between single and double lung images is significantly higher in doctors [staff \& residents] than in non-doctors [others]. Thus, Expertise \& CS information plays a significant role in the diagnosis of pneumoconiosis. CS information helps in diagnosing pneumoconiosis by reducing the general tendency of giving less profusion ratings. Training and experience appear to play important roles in learning to use the CS information present in the chest radiographs.
\end{abstract}

Keywords: Pneumoconiosis, Chest radiographs, Eye tracking study, Contralateral symmetry, Radiological training and expertise, Lung zones, Fixation time

\section{INTRODUCTION}

There are millions of people around the world, working in industrial areas. Pneumoconiosis is the inflammation of lungs caused by repeated exposure to small particles of industrial dust that enters the lungs ${ }^{1}$. There have been some impressive technical advances in the diagnosis of lung diseases during the last 25-30 years, but the chest radiograph remains the most common and widely used tool. Chest radiograph is still ubiquitous in clinical practice, and will likely remain so for quite some time ${ }^{8}$. Diagnosis of this disease is done through radiological observation of chest radiographs. The diagnosis of pneumoconiosis is a complex procedure and it requires a certain level of expertise ${ }^{2,4}$. Some studies proved that there is significant inter reader and some intra reader variation in the diagnosis of pneumoconiosis ${ }^{3,4}$. So, in general, there will be a hierarchy of readers for diagnosis of this disease. ILO has also introduced a standard

${ }^{1}$ varunjampani@research.iiit.ac.in; Phone: +91-7799595300 
classification scheme for the diagnosis of pneumoconiosis to facilitate the international comparisons of data, epidemiological investigations and research reports ${ }^{5}$. The details of ILO classification scheme are explained elsewhere ${ }^{5}$.

Diagnosis of pneumoconiosis seems to be very subjective as there is substantial inter-reader variation and some intra-reader variation ${ }^{4}$. The reader consistency from one time period to another is felt to be within acceptable limits, provided the reader is experienced. From the above discussion, we can say that expertise plays a very important role in the diagnosis of pneumoconiosis. Even though there are studies ${ }^{2,3,4,9}$, showing the importance of expertise, to our knowledge, there are no studies on how the different levels of expertise affect the diagnosis of the disease.

One of the aims of the present study is to investigate the role of Contralateral Symmetry (CS) present in chest radiographs, in diagnosis of Pneumoconiosis. Symmetry is present in many of the objects we encounter in our daily lives. The detection of symmetry is one of the characteristics of human visual perception. This may be due to the abundant examples of symmetry in the structure and development of the human and animal forms. For example, symmetry plays a very important role in visual processes such as face perception ${ }^{6}$ and attractiveness ${ }^{7}$. Much of the gross anatomy of the human body also exhibits contra-lateral symmetry. Symmetry seems to be playing a major role in perception of things around us.

PA Chest X-rays also exhibit good amount of CS. Strictly speaking, lungs are naturally positioned in pseudocontralateral symmetry, but the near perfect contralateral symmetrical occurrence of abnormal lung tissue in both lungs is highly improbable. There are very good chances that radiologists use this contra-lateral symmetric information present in chest X-rays while reading them. It has been emphasized in the radiological training that the best way to read any chest X-ray, of any disease, is to compare the left and right lung zones.

Observer studies have shown the potential usefulness of contralateral subtraction ${ }^{11,12,13}$, a technique that employs the symmetry of the rib cage to subtract one side of the lung from the other side, so that the abnormalities stand out more clearly. This technique has been used for improving the detection accuracy of pulmonary nodules ${ }^{14}$. Despite the existence of some observer studies on the usefulness of contra-lateral subtraction technique, to our knowledge, there are no empirical studies on the role of CS in diagnosing lung diseases. Moreover, unlike lung nodules (which are localized), pneumoconiosis is a diffused lung disease. Hence it is likely that CS plays even a bigger role in diagnosing such a diffuse disease.

The main aim of our experiment is to study the role of CS and expertise in the diagnosis of pneumoconiosis. Specifically, we wish to understand the beneficial and detrimental effects of using CS information present in chest radiographs in the diagnosis of pneumoconiosis. The role of CS can be studied by presenting single lung vs. double lung images for reading. A good way to understand the role of expertise is to study the variability in reading pattern across subjects ranging from novice to experts. Eye tracking is the best way to extract information on the reading pattern and hence is our chosen method of study.
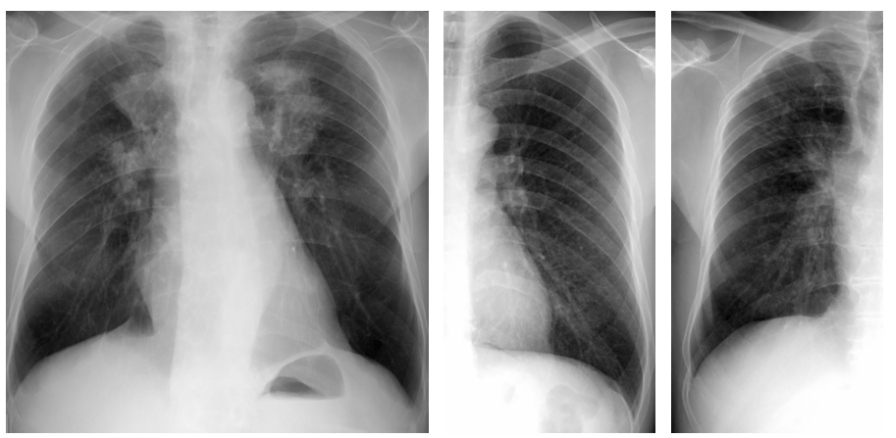

Figure 1: Sample double (left) and single (right) lung images used in the experiment 


\section{METHODS}

Our experiments were conducted in a room, dedicated to eye tracking experiments. Written informed consent, for the study and eye movement recording, was taken from all the participants. Patient informed consent for the use of the chest X-rays that were collected between 2006 and 2008 was not required. The chest X-rays, used for the experiment, were provided by the Shanghai Pulmonary Hospital, China.

\subsection{Test Images}

The test image set consisted of 33 PA digital chest X-rays, of which 17 were normal, full, double-lung images and 16 were single-lung images with only one lung. Figure 1 shows sample single- and double-lung images. Test images included images of disease stages: 1, 2 and 3. Table-1 shows the detailed composition of the test set.

Table 1: Images of different disease stages used in our experiment

\begin{tabular}{|c|c|c|}
\hline Disease stage & Double Lung Images & Single Lung Images \\
\hline Stage 1 & 3 & 2 \\
\hline Stage 2 & 4 & 6 \\
\hline Stage 3 & 10 & 8 \\
& Total: 17 & Total: 16 \\
\hline
\end{tabular}

The above X-ray images were of workers working in an industrial environment with either silica or metal dust. The workers had spent anywhere between 2 years and 31 years $(M d n=12)$ in this environment. The experienced radiologists at Shanghai Pulmonary Hospital, China provided ground truth for these images.

\subsection{Participant Details}

Since one of the purposes of our experiment was the study of role of expertise, we ran the experiment on subjects varying from novices and undergraduate medical students to expert radiologists. A total of 23 participants volunteered for the experiment. These participants were in 4 categories: staff radiologists (4), resident radiologists (4), medical students $(3 / \mathrm{yr}$ x 4 yrs $=12)$ and 3 novices (3). We will, in the course of our discussion, refer to the members of the first two categories put together as doctors and the last two as non-doctors. The staff radiologists have an experience ranging from 11 years to 58 years $(M=30.75)$. Resident radiologists had at least 3 years of training.

\subsection{Experimental Procedure}

We used with-in subjects design to study the role of CS present in chest X-rays. Accordingly, each participant was shown both single- and double-lung images. The entire test set of 33 images was shown to each participant, in random order. Each lung region was divided into 3 zones: upper, middle and lower. The task given to a participant was to report the profusion level of each zone in written form. Information such as localization of anomalies, their size and shape are not asked, as the profusion level categorization is of primary importance to clinical settings. Since none of the 
participant had any expertise in pneumoconiosis, we used $4(0,1,2$ and 3$)$ levels of profusion categorization rather than 12 levels used in ILO standards.

The participant's gaze was tracked using remote/head-free eye tracker (Model Eyelink $1000^{10}$, SR research, Canada). Participants could freely move their heads while viewing the chest X-rays, as in clinical settings. Before the beginning of the experiment, all the subjects were given training in which the concept of profusion level was explained using some sample chest X-rays. The procedure for dividing each lung field into zones was also explained in this training session. A 9-point camera calibration was done to track eye movements. Experimental images were shown one after the other to every subject. Unlimited time was given to view each image and report profusion ratings for all the 6 zones of the lungs. Eye movement data, response times and profusion ratings were recorded for each subject and for each image.

\section{RESULTS}

\subsection{Analysis of observers' diagnostic performance}

Different observer studies ${ }^{2,4,15}$ on the diagnosis of Pneumoconiosis have used different performance measures to quantify the diagnostic performance. In our present study, instead of using a single measure, we used different performance measures to evaluate the observer error. Sum of absolute differences (explained below) is taken as primary measure of observer error in our analysis. Since, this measure fails to capture all aspects of the observer performance, we have also used penalize over and penalize under.

The observer error is obtained by taking the average sum of absolute differences between the profusion ratings, for each lung, and the ground truth profusion values as follows.

$$
\text { Observer Error, } o=\frac{1}{n} \sum_{i=1}^{n}|r-p|
$$

Where $n$ : Total number of Zones

$p$ : Ground truth profusion rating

$r$ : Participant's (observer's) profusion rating

As self-evident, this performance measure is directly proportional to the observer error. Figure-2 shows plots of $o$ for participant groups of different expertise levels, for both double and single lung images.

Some observations can be made from these plots. The observer error $o$ (grey bars) for double lung images is seen to vary significantly with expertise, which was confirmed by Kruskal-Wallis test $\left(\chi^{2}(6)=13.38, p=.038\right)$. The error for single-lung images is consistently higher than for double-lung images across the different categories, with the differential being higher as expertise increases. The latter trend was confirmed by the Mann-Whitney test $(U=28, p=.038)$ according to which the error $M d n$ is 0.38 for doctors while it is only 0.18 for non-doctors. For double-lung images, there is a decrease in error with increase in expertise; however, this trend was not present in the case of single-lung images.

It was seen from Wilcoxon-signed rank test that the observer error $(M d n=0.813)$ was significantly higher for single-lung images $(Z=3.13, p<.001)$ than for the double-lung images $(M d n=0.620)$ for all the participants. Based on these results, it can be concluded that CS information plays an important role in the diagnosis of pneumoconiosis and it is perhaps used most effectively by doctors. Next, we did finer analysis to see how CS affects the actual profusion rating across different expertise levels. 

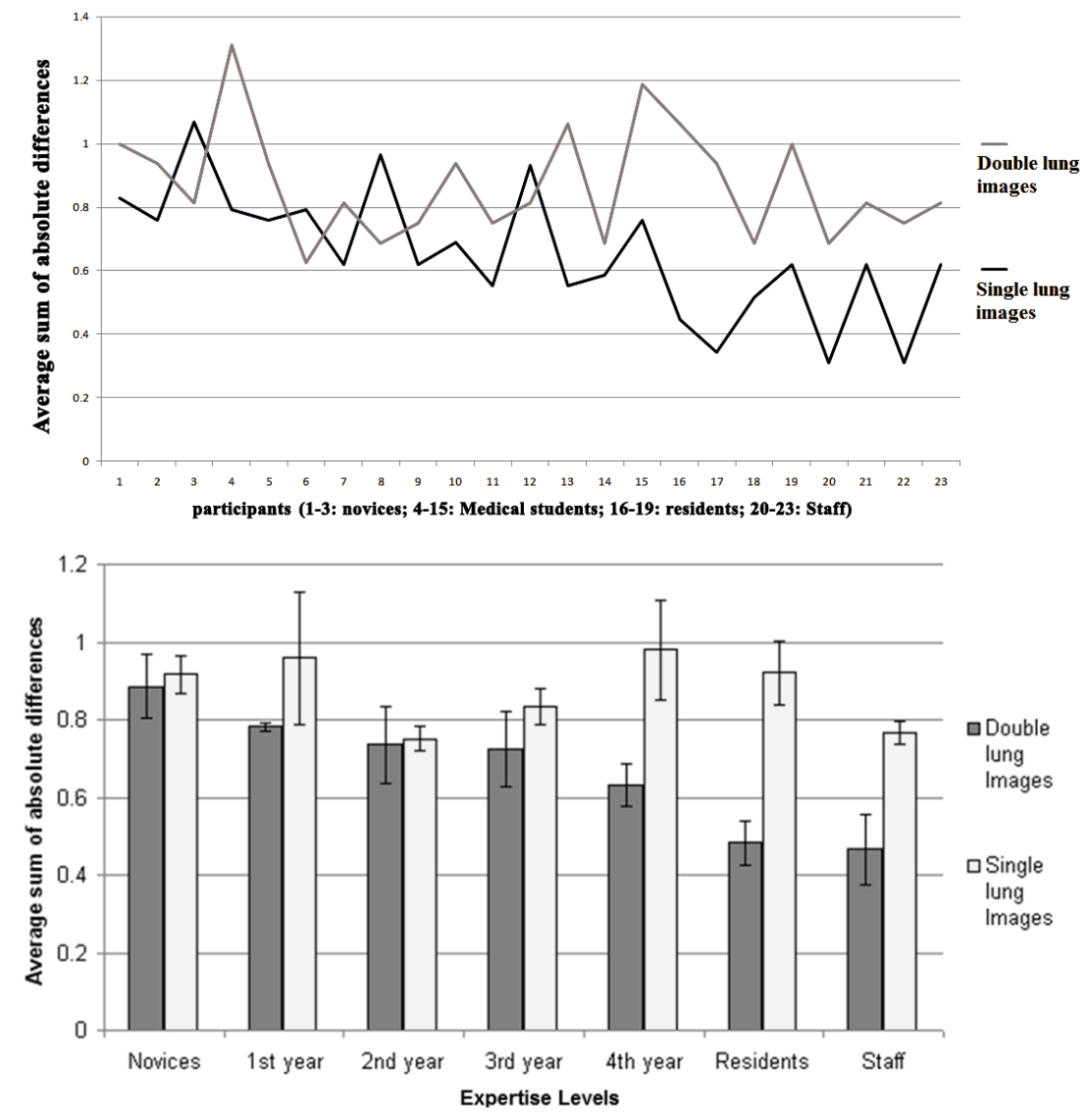

Figure 2: Performance of different expertise groups for both single and double lung images. Above: Graph of the average sum of absolute differences for all participants. Below: Bar graph of the average sum of absolute differences.

Penalize Over/Under are the total number of times a participant has given a profusion rating higher/lower than that of ground truth profusion value. Figure 3 shows the average penalize over and average penalize under values, for participant groups of different expertise levels.

From Figure on the left, it can be seen that except, the staff radiologist group, observers tend to penalize over more for double- rather than single- lung images. More generally, when considering all the participants, the Wilcoxon signed-rank test revealed that there is more penalize over $(Z=2.13, p=.033)$ in double-lung images $(M d n=0.31)$ than in single lung images $(M d n=0.25)$. Figure-3 (right) shows more average penalize under in single lung images than in double lung images. When considering all the participants, Wilcoxon signed rank test revealed that there is indeed more penalize under $(Z=3.89, p<.001)$ in single lung images $(M d n=0.34)$ than in double lung images $(M d n=0.28)$.

From the above analysis, it appears that observers give a higher rating to a zone when the contra-lateral region is available for comparison. Hence, it can be concluded that CS has a role not only in correctly diagnosing pneumoconiosis but also in gauging the severity i.e. assigning profusion ratings. 

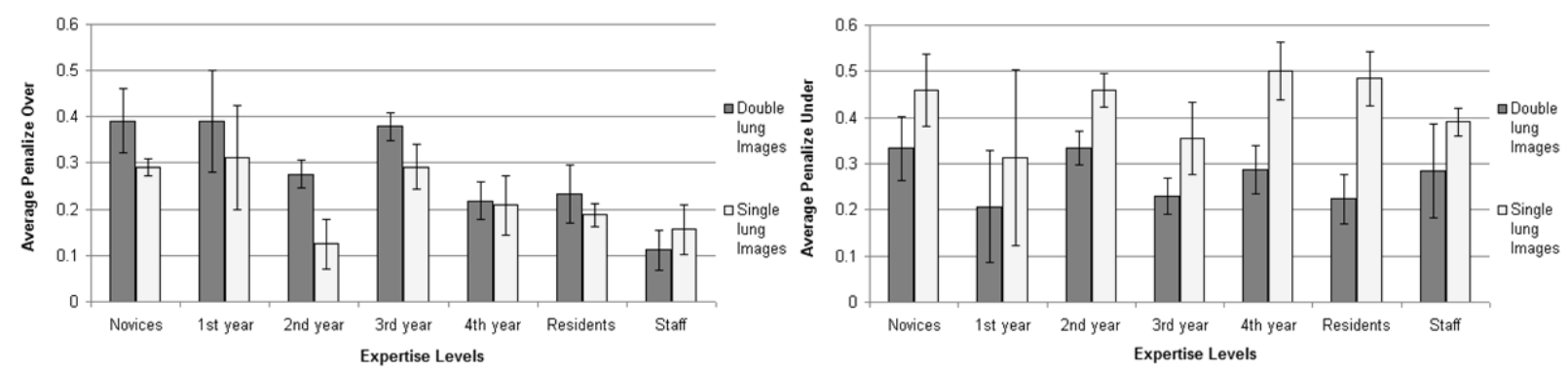

Figure 3: (left) Average penalize over and (right) average penalize under, for different expertise groups.

\subsection{Eye movement Analysis}

Figure-4 shows sample heat maps on a double and 2 single lung images for a staff radiologist. In double lung images, analysis showed that observers spent almost same amount of time in viewing both lungs pointing to their use of CS in diagnosis. Analysis of eye movements showed a good correlation between observer error and time spent in viewing. Specifically, observers spent less fixation time in the zones they considered to be clearly normal and clearly abnormal (profusion levels 0 or 3 ) resulting in high observer error. Detailed results are not presented here as they are out of scope of this paper.
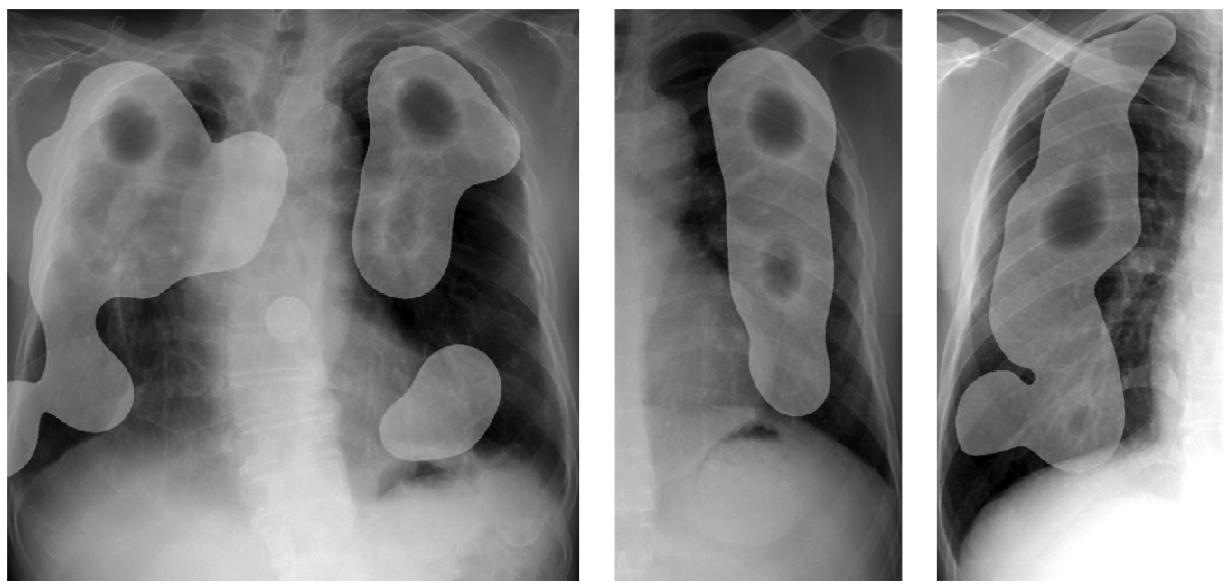

Figure 3: Sample heat maps showing eye fixation density of a staff radiologist on a double (left) and single (right) lung images

\section{DISCUSSION}

The aim of the present study is the study of the role of CS on the diagnosis of pneumoconiosis and also its influence on the readers with different expertise levels. Analysis of observer error (average sum of absolute differences) indicated that contralateral symmetry plays a significant role in the diagnosis of pneumoconiosis and its role is more 
important in the case of doctors than in the case of non-doctors. This shows that training and experience plays a very important role in learning how to use the contralateral symmetric information present in the chest radiographs.

The analysis of 'penalize over' and 'penalize under' gave some indications for how this contralateral symmetric information present in the chest radiographs is helping in correctly estimating the profusion in a zone. Analysis showed that observers give more rating to a zone when they have other side of lung to compare with, than not. Giving more rating may have both positive and negative effects, depending upon the true profusion value. One of the previous studies

${ }^{16}$ showed that application of ILO classification could result in roentgenographic underestimation of asbestosis (a variant of pneumoconiosis). Our results also showed that there is general tendency to give less profusion rating, in the case of staff radiologists. Our analysis showed that this tendency of giving less profusion rating increases when there is no contralateral symmetric information available to the reader, leading to more observer error. Thus, contralateral symmetric information present in the chest radiographs helps in diagnosing the pneumoconiosis by reducing the tendency of giving less profusion ratings.

Even though our study showed that contralateral symmetric information present in the chest X-rays helps in diagnosing pneumoconiosis, we still don't know how this contralateral symmetric information is helping in diagnosis. In more precise terms, we still don't know, at what level this contralateral symmetric information is useful i.e. at image level or zonal level or at intra-rib region level. At this point, we can't make any conclusions regarding this. More experiments are needed to study these more detailed aspects of contralateral symmetry and how these are affecting the diagnosis.

Analysis of eye movements showed that zones where the observers made high absolute error (3) are zones where less time was spent in viewing. This indicates the importance of time in the diagnosis of pneumoconiosis. A clear decrease in fixation time, with increase in observer error (except in the case of 0 and 1), in single lung images, suggests that time has a very important role to play in single lung images. Less fixation time in the zones which observers think as clearly normal and clearly abnormal, and less fixation time in the zones of high observer error indicates that zones are needed to be looked more carefully even when the observer thinks it as clearly normal or abnormal. In other words, for better diagnostic results, all the zones should be looked carefully i.e. X-rays should not be speed-read.

\section{CONCLUSION}

This article studies the role of expertise and contra-lateral Symmetry in the diagnosis of Pneumoconiosis via a human experiment. Results indicated that Expertise and CS play important roles in the diagnosis of pneumoconiosis. A key finding of our study is that the presence of CS information alone does not help improve diagnosis as much as learning how to use the information. This learning appears to be gained from focused training and years of experience. Hence, good training for radiologists and careful observation of each lung zone may improve the quality of diagnostic results.

Further experiments are required to determine the exact role of CS in diagnosing chest radiographs i.e. how exactly this information is being used by the radiologists. Since our experiment involves only the chest radiographs of pneumoconiosis, which is a diffused lung disease, the results may not be applicable to localized lung diseases such as lung cancer etc. These issues remain the topic of future work.

\section{ACKNOWLEDGMENTS}

The authors would like to thank Bipin Indurkhya, Ph.D, for helpful discussions and his assistance in our experimental design. We would like to express our appreciation to Peter Ajemba, Ph.D, for his assistance in data analysis and Kakarla Subba Rao, MD, for his helpful discussions regarding the reading of chest radiographs. 


\section{REFERENCES}

[1] Mason, R.J., Murray, J.F., Boraddus, V.C. and Nadel, J.A., [Textbook of Respiratory Medicine], Saunders Elsevier, $4^{\text {th }}$ ed. Philadelphis, 1758-1763, (2005).

[2] Morgan, R.H., Donner, M.W., Gayler B.W., Margulis, S.I., Rao, P.S. and Wheeler, P.S., "Decision processes and observer error in the diagnosis of pneumoconiosis by chest roentgenography", AJR, 117, 757-764 (1973).

[3] Kruger, R.P., Thompson, W., and Turner, A., "Computer diagnosis of pneumoconiosis", IEEE Trans. Syst., Man, Cybern., vol. SMC-4, p. 40 (1974).

[4] Reger, R.B., Smith, C.A., Kibelstis, A., and Morgan, K.C., "The effect of film quality and other factors on roentgenographic categorization of coal workers pneumoconiosis", Amer. J. Roetgenol. Radum Ther. Nucl. Med., 115, 462-472 (1972).

[5] I.L. Office, "Guidelines for the use of ILO international classification of radiographs of pneumoconiosis", International Labour office: Geneva (1980).

[6] Chen, C.C., Kao, K.L. and Tyler, C.W., "Face configuration processing in the human brain: the role of symmetry", Cerebral Cortex., 17(6), 1423-1432 (2007).

[7] Grammer, K. and Thornhill, R., "Human (Homo sapiens) facial attractiveness and sexual selection: the role of symmetry and averageness", J Comp Psychol., 108(3), 233-42 (1994).

[8] Ginneken, B., Romeny, B.H., and Viergever, A., "Computer-Aided diagnosis in Chest Radiography: A survey", IEEE transactions on medical imaging, 20, No. 12 (2001).

[9] Pearson, N.G., Ashford, J.R., Morgan, D.C., Pasqual, R.S.H. and Rae, S., "Effect of quality of chest radiographs on categorization of coal workers' pneumoconiosis". British Journal of Industrial Medicine, 22, 81-92 (1965).

[10] http://www.sr-research.com/EL_1000.html

[11] Li, Q., Katsuragawa, S., Ishida, T., Yoshida, H., Tsukuda, S., MacMahon, H., and Doi, K., "Contralateral subtraction: A novel technique for detection of asymmetric abnormalities on digital chest radiographs", Med. Phys., 27(1), 47-55 (2000).

[12] Yoshida, H. and Doi, K., "Computerized detection of pulmonary nodules in chest radiographs: reduction of false positives based on symmetry between left and right lungs", Proc. SPIE., 3979, 97 (2000).

[13] Li, Q., Katsuragawa, S. and Doi, K., "Improved contralateral subtraction images by use of elastic matching technique", Med. Phys., 27(8), 1934-1942 (2000).

[14] Tsukuda, S., Heshiki, A.,Katsuragawa, S., Li, Q., MacMahon, H., and Doi, K., "Detection of Lung Nodules on Digital Chest Radiographs: Potential Usefulness of a New Contralateral Subtraction Technique", Radiology, 223, 199203 (2002).

[15] Liddell, F.D.K., “An experiment in film reading”, Brit. J. Industr. Med., 20, 300, (1963).

[16] Rockoff, S.D., Schwarz, A., "Roentgenographic underestimation of early asbestosis by International Labor Organization classification. Analysis of data and probabilities", Chest, 96(4),952 (1989). 